\title{
Effects of nontransmural ischemia on inner and outer wall thickening in the canine left ventricle
}

\begin{abstract}
The effect of ischemic subendocardial dysfunction on contractile function in the normally perfused subepicardium remains controversial. Accordingly, regional wall thickening (WT) was measured directly in the left ventricle of 10 open-chest dogs using epicardial echocardiography. Two silk sutures, used as echocardiographic targets, were inserted beneath the transducer to a depth of $25.0 \pm 0.7 \%$ (subepicardium) and $48.0 \pm 2.7 \%$ (midmyocardium) of transmural thickness. A hydraulic cuff, placed around the left anterior descending coronary artery (LAD) was then inflated slowly until transmural WT was reduced to $62 \pm 2 \%$ of baseline. Myocardial blood flow (MBF) was not significantly altered in the subepicardial third of the wall; however, flow to the midwall and subendocardial thirds decreased by $39 \%(p<0.001)$ and $50 \%(p<0.001)$, respectively. Nontransmural ischemia produced a small but significant decrease in epicardial WT (baseline $=0.77 \pm 0.08 \mathrm{~mm}$, ischemia $=0.69 \pm 0.08 \mathrm{~mm} ; \rho<0.05$ ) and substantially larger decreases in midwall (baseline $=1.66 \pm 0.14 \mathrm{~mm}$, ischemia $=1.03 \pm 0.09 \mathrm{~mm} ; p<0.001$ ) and subendocardial WT (baseline $=3.39 \pm 0.34 \mathrm{~mm}$, ischemia $=2.10 \pm 0.26 \mathrm{~mm} ; p<0.001$ ). The degree of regional dysfunction was linearly correlated with tissue depth $(r=0.88, p<0.001)$. Thus the degree of dysfunction produced by nontransmural ischemia increased progressively from the subepicardium to the subendocardium, paralieling the pattern of perfusion. We conclude that perfusion, rather than transmural tethering, largely determines subepicardial function in the setting of nontransmural ischemia. (AM HEART J 1991;122:1292.)
\end{abstract}

Ronald J. Torry, PhD, J. Hurley Myers, PhD, Andrew L. Adler, MS, Christine L. Liut, BS, and Kim P. Gallagher, PhD. ${ }^{a}$ Carbondale, Ill., and Ann Arbor, Mich.

In a previous study, we described an echocardiographic technique that enables direct measurement of transmural differences in wall thickening dynamics. ${ }^{1}$ A suture was passed through the myocardial wall that could be visualized with an epicardial echocardiographic transducer. Utilizing this technique, we demonstrated that inner wall thickening was greater than outer wall thickening during control conditions, consistent with theoretical predictions and studies using other methods..$^{2-7}$ The objective of the present study was to use the epicardial echocardiographic technique to determine the effects of par-

\footnotetext{
From the Department of Physiology, School of Medicine, Southern Illinois University, Carbondale; and the Thoracic Surgery Research Laboratory, Departments of Surgery and Physiology, The University of Michigan Medical School, Ann Arbor.

Supported in part by a grant-in-aid from the Illinois Affiliate of the American Heart Association, by National Institutes of Health grant R01 H132043, and by National Institutes of Health Research Career Development Award K04 HL01420.

Received for publication Dec. 18, 1990; accepted May 24, 1991.

Reprint requests: J. H. Myers, PhD, School of Medicine, Southern Illinois University, Carbondale, IL 62901 .

$4 / 1 / 32180$
}

tial coronary occlusion on transmural differences in wall thickening dynamics.

Previous studies have demonstrated that a partial coronary occlusion results in a redistribution of coronary blood flow. Blood flow to the subepicardium can remain at normal levels when flow to the subendocardium is greatly reduced due to the different autoregulatory pressure limits in the inner and outer myocardial layers. ${ }^{8}, 9$ The functional effects of nontransmural ischemia have been addressed in studies in which epicardial segmental shortening was used as a parameter of outer wall function,,$^{10-15}$ but largely contradictory results have been obtained. The alignment of sonomicrometers with respect to epicardial fiber orientation and regional differences within the left ventricle may explain some of the variability among these studies. By measuring wall thickening rather than epicardial segment shortening, we attempted to evaluate outer wall contractile function without the potential problems posed by gauge alignment with respect to fiber orientation.

\footnotetext{
METHODS

Experimental preparation. Ten mongrel dogs (24.6 $\pm 4.2 \mathrm{~kg}$, mean $\pm \mathrm{SD}$ ) were premedicated with fentanyl
} 
citrate (Innovar-Vet, Janssen Pharmaceutica, Inc., Piscataway, N.J., 0.11 to $0.14 \mathrm{ml} / \mathrm{kg}$, intramuscularly), then anesthetized with alpha-chloralose $(120 \mathrm{mg} / \mathrm{kg}$, intravenously). Anesthesia was maintained throughout the experiment by an intravenous infusion of chloralose $(80 \mathrm{mg} /$ $\mathrm{kg} / \mathrm{hr}$ ). The dogs were intubated and ventilated with a Harvard respirator (Harvard Apparatus Inc., S. Natick, Mass.). A left thoracotomy was performed through the fifth intercostal space and the heart was suspended in a pericardial cradle to maximize exposure of the anterior free wall of the left ventricle. A segment of the left anterior descending coronary artery distal to the first major diagonal branch was exposed by dissection and a hydraulic cuff coupled to a Harvard infusion pump (Harvard Apparatus Inc.) was placed around the artery. A Konigsberg high-fidelity micromanometer (Model P7, Konigsberg Instruments, Inc., Pasedena, Calif) and a fluid-filled Tygon catheter (Norton Plastics, Akron, Ohio) (used to calibrate the micromanometer in millimeters of mercury) were inserted into the left ventricle via the left atrial appendage. A catheter was also inserted into the left atrium to serve as an injection site for tracer-labeled microspheres. In five dogs, a bipolar pacing catheter was inserted into the right atrium and was secured with purse string sutures. Catheters were also placed in the femoral artery to enable measurement of arterial blood pressure and to obtain reference arterial blood samples for calculation of regional myocardial blood flow. 'The femoral vein was cannulated to provide access for intravenous fluids and alpha-chloralose.

Measurement of regional myocardial thickening. A 6 $\mathrm{mm}, 5 \mathrm{MHz}$, nonfocused epicardial echocardiographic transducer (KB-Aerotech, Lewistown, $\mathrm{Pa}$.) coupled to an Irex-M-mode echocardiographic recorder (Model 101, Irex Meridian, Johnson \& Johnson, New Brunswick, N.J.) was used to measure regional wall thickening. The transducer was attached directly to the epicardium with cyanoacrylate adhesive and was oriented to obtain a strong continuous signal from the endocardium, as previously described. ${ }^{1}$ The flexible transducer cable was supported by an adjustable arm suspended from a ring stand and was positioned to allow the transducer to "ride" with the heart through each cardiac cycle with minimal torsional movement. Myers et al. ${ }^{1}$ have demonstrated that regional wall motion is not compromised as a result of the attachment of the transducer directly to the surface of the heart.

For direct measurement of transmural differences in wall thickening, two waxed, 3-0 silk sutures were inserted into the myocardial wall directly beneath the echocardiographic transducer using curved surgical needles. ${ }^{1}$ One suture was placed in the subepicardial portion of the left ventricular wall (average depth from epicardial transducer interface $=25.0 \pm 0.7 \%$ of transmural thickness; range $=20.7 \%$ to $27.9 \%$ ) and the other suture was placed approximately midway between the endocardium and epicardium (depth from epicardial surface $=48.0 \pm 2.7 \%$; range $=38.0 \%$ to $64.5 \%$ ). The sutures and endocardium were visualized simultaneously, thereby allowing direct measurement of subepicardial (outer quarter), midwall (outer half), and subendocardial (inner half) thickening along the same ra-
Table I. Hemodynamic variables during baseline and partial coronary occlusion

\begin{tabular}{lcc}
\hline & Baseline & PCO \\
\hline Heart rate (beats/min) & $107 \pm 9$ & $108 \pm 8$ \\
Systolic BP (mm Hg) & $118 \pm 3$ & $117 \pm 4$ \\
Diastolic BP (mm Hg) & $81 \pm 4$ & $77 \pm 3$ \\
Mean BP (mm Hg) & $94 \pm 3$ & $91 \pm 3$ \\
+dP/dt (mm Hg/sec) & $2047 \pm 118$ & $1840 \pm 117^{*}$ \\
-dP/dt (mm Hg/sec) & $1890 \pm 118$ & $1708 \pm 106$ \\
LVEDP (mm Hg) & $5.8 \pm 0.7$ & $6.8 \pm 1.1$ \\
\hline
\end{tabular}

Data presented as means $\pm \mathrm{SEM} ; n=10$.

PCO, Partial coronary occlusion; BP, blood pressure; LVEDP, left ventricular end-diastolic pressure.

${ }^{*} p<0.05$ from control (paired $t$ test)

dial axis through the anterior wall. The echocardiographic data were recorded on rapid process photographic paper for later analysis. The reflected ultrasound was amplified so that $10 \mathrm{~mm}$ of muscle thickness equalled $33 \mathrm{~mm}$ on paper. Measurements were made to the nearest $0.5 \mathrm{~mm}$ on paper $(0.15 \mathrm{~mm}$ muscle) and were averaged over 5 to 10 cardiac cycles.

Wall thickening measurements were made as described previously. ${ }^{1}$ Briefly, the variables analyzed included: (1) wall thickness at end diastole (identified at the peak QRS complex of the ECG); (2) end-systolic wall thickness at maximum thickness; (3) systolic wall excursion (endsystolic thickness minus end-diastolic thickness); (4) percentage wall thickening (systolic wall excursion divided by diastolic thickness $\times 100$ ); and (5) the fractional contribution of subepicardial, midwall, and subendocardial thickening to total transmural thickening (wall excursion divided by the transmural excursion $\times 100$ ).

A limb lead electrocardiogram was monitored continuously to verify normal rhythm and to measure heart rate. Left ventricular peak systolic and end-diastolic pressures were measured with the Konigsberg micromanometer (Konigsberg Instruments, Inc.). The first derivative of the left ventricular pressure signal was obtained with a Beckman R-C differentiation circuit (Beckman Instruments Inc., Fullerton, Calif.). The differentiator was calibrated prior to each study by using an input signal with a known slope. Aortic systolic, diastolic, and mean pressures were measured with fluid-filled Tygon tubing (Norton Plastics) coupled to Statham P23Db pressure transducers (ViggoSpectramed Inc., Critical Care Division, Oxnard, Calif.). Analog signals were recorded simultaneously on an eightchannel Beckman R-611 Dynagraph (Beckman Instruments Inc.) and a Hewlett-Packard FM tape recorder (Model 3968A, Hewlett-Packard Co., Medical Products Group, Andover, Mass.).

Measurement of regional myocardial blood flow. Regional myocardial blood flow was measured with microspheres (15 $\pm 3 \mu \mathrm{m}$ diameter, New England Nuclear, Dupont, North Billerica, Mass.) labeled with either ${ }^{141} \mathrm{Ce}$, ${ }^{95} \mathrm{Nb},{ }^{46} \mathrm{Sc}$, or ${ }^{103} \mathrm{Ru}$, using the reference withdrawal technique. ${ }^{16}$ Approximately one to two million microspheres 


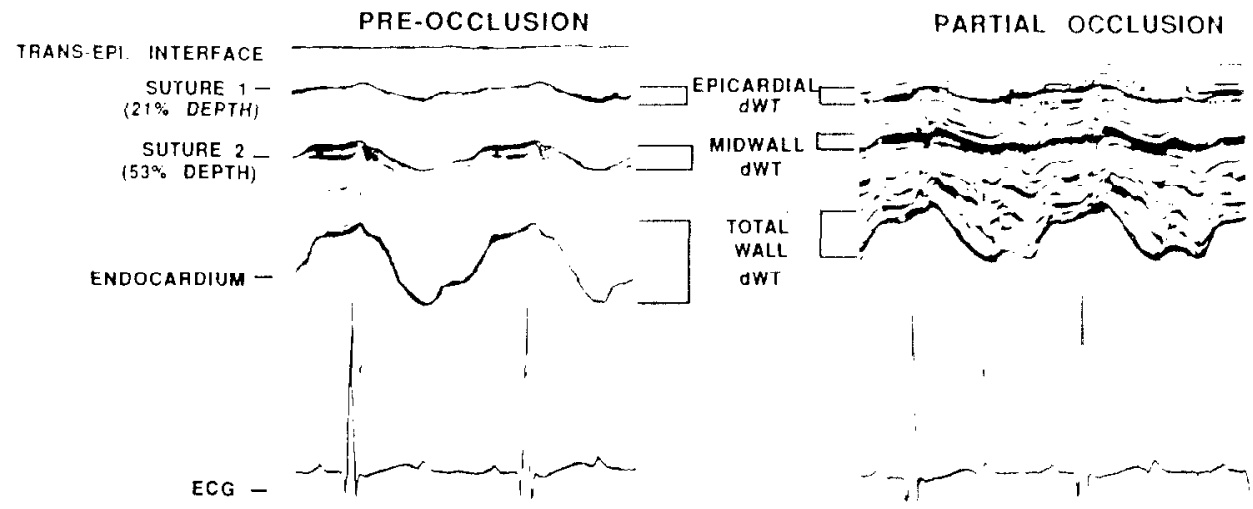

Fig. 1. Sample echocardiogram from a dog with two sutures inserted in the myocardium. The left panel illustrates regional transmural thickening during preocclusion (i.e., control), while the right panel illustrates regional transmural thickening 5 minutes after a partial coronary occlusion is produced in the same animal. See text for details. $d W T$, Difference in wall thickness from diastole to systole (i.e., wall excursion); Trans-epi. interface, transducer-epicardial interface.

Table II. Regional myocardial wall thickness data during baseline and partial coronary occlusion

\begin{tabular}{lrrrr}
\hline & $E D W T(\mathrm{~mm})$ & $E S W T(\mathrm{~mm})$ & $d W T(\mathrm{~mm})$ & $\% d W T$ \\
\hline Baseline conditions & & & & $30.8 \pm 3.7$ \\
EPI & $2.56 \pm 0.11$ & $3.33 \pm 0.12$ & $0.77 \pm 0.08$ & $34.0 \pm 2.3$ \\
MID & $4.90 \pm 0.32$ & $6.56 \pm 0.43$ & $1.66 \pm 0.14$ & $49.5 \pm 2.3$ \\
TOTAL & $10.26 \pm 0.47$ & $15.33 \pm 0.71$ & $5.06 \pm 0.31$ & $28.7 \pm 3.6$ \\
Partial coronary occlusion & $2.44 \pm 0.09^{*}$ & $3.13 \pm 0.10^{*}$ & $0.69 \pm 0.08^{*}$ & $21.9 \pm 1.8^{*}$ \\
EPI & $4.76 \pm 0.29^{*}$ & $5.79 \pm 0.34^{*}$ & $1.03 \pm 0.09^{*}$ & $30.9 \pm 1.4^{*}$ \\
MID & $10.11 \pm 0.46$ & $13.24 \pm 0.65^{*}$ & $3.13 \pm 0.22^{*}$ & \\
TOTAL & & &
\end{tabular}

Data presented as means $\pm \operatorname{SEM}(n=10)$.

EPI, Subepicardial quarter of the left ventricular wall (outer $21^{\prime} ;$ to $28 \%$ of wall thickness); MID, outer half of the left ventricular wall (outer $38^{\circ}$ to $65^{\circ} ;$ of wall thickness); TOTAL, transmural wall thickness; EDWT, end-diastolic wall thickness; ESWT, end-systolic wall thickness; dWT, systolic wall thickening excursion (ESWT - EDWT); $\approx \mathrm{dWT}$, percentage systolic wall thickening (dWT/EDWT $\times 100)$

${ }^{*} p<0.05$ compared with control value (paired $t$ test).

were injected into the left atrial catheter and were flushed with $15 \mathrm{ml}$ of saline over a 30 -second period. The arterial reference sample was withdrawn at a constant rate $(7.5$ $\mathrm{ml} / \mathrm{min}$ ) beginning prior to microsphere injection and continuing for 90 seconds thereafter. At the end of each study, the hearts were removed and were placed in formalin for 3 to 4 days to facilitate sectioning. Full thickness tissue samples were taken from the ischemic zone (beneath the echocardiographic transducer) and the normally perfused (control) zone. Each sample was divided into three pieces of approximately equal thickness from endocardium to epicardium. After corrections for overlap counts, myocardial blood flow to each "layer" was calculated using standard methods. ${ }^{16}$ To minimize the variability in blood flow from $\operatorname{dog}$ to $\mathrm{dog}$, blood flow in the ischemic zone was expressed as a percentage of nonischemic zone flow, similar to the convention used in other studies involving measurements of regional myocardial blood flow with microspheres. ${ }^{13,17-20}$

After baseline echocardiographic and hemodynamic variables were recorded (at least 30 minutes after instrumen- lation was completed), the first set of radioactive microspheres was injected. The hydraulic occluder was then inflated slowly $(0.382 \mathrm{ml} / \mathrm{min})$ until a $40 \pm 10 \%$ decrease in transmural systolic excursion was achieved. The stenosis was maintained for at least 3 to 5 minutes, then recordings of wall thickness and hemodynamic variables were repeated and a second set of microspheres was injected. A minimum of 3 minutes was chosen before sampling to permit enough time for vascular resistance distal to the stenosis to adjust to the reduction in perfusion pressure. ${ }^{8}$

To verify that data obtained after 3 to 5 minutes of partial coronary occlusion represented a steady-state condition, five dogs were paced from the right atrium at a rate 8 to 10 beats/min greater than their intrinsic rate $(123 \pm 3$ beats/min), and the partial coronary occlusion was sustained for 1 hour. The occluder was adjusted as needed to maintain a constant decrease in transmural excursion over the time period. ${ }^{18}$ Hemodynamic and echocardiographic data were recorded and a third and fourth set of microspheres were injected at 30 and 60 minutes of nontransmural ischemia. 


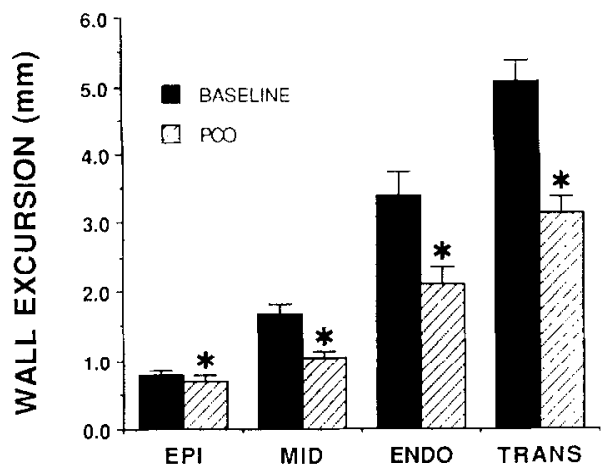

Fig. 2. Regional changes in wall thickening during partial coronary occlusion ( $P C O$ ). EPI, Subepicardial suture data; $M D$, midwall suture data; $E N D O$, transmural minus midwall data; TRANS, transmural data. ${ }^{*} p<0.05$ (paired $t$ test).

To terminate each experiment, the dog was given an overdose of chloralose and ventricular fibrillation was induced. This method of euthanasia is consistent with the recommendation of the Panel on Euthanasia of The American Veterinary Medical Association. The heart was removed, fixed in $10 \%$ buffered formalin, and was later sectioned for determination of regional myocardial blood flow.

Unless otherwise stated, all values are expressed as means \pm standard error of the mean. Significant differences between baseline and inflow restriction values for the echocardiographic, hemodynamic, and regional blood flow data were determined using Student's $t$ test for paired data. In the experiments with 60-minute sustained partial occlusions, $a$ one-way analysis of variance for repeated measures was employed to determine statistically significant differences between means over time. When overall significance was detected, a Dunnett's $t$ test was used to detect differences between time points. For all tests, statistical significance was indicated when $p<0.05$.

\section{RESULTS}

Hemodynamic data during baseline conditions and partial coronary occlusion are summarized in Table I. There were no significant changes in heart rate, arterial blood pressures, or left ventricular end-diastolic pressure during partial coronary occlusion. Positive dP/dt, however, decreased significantly during the partial occlusion.

Fig. 1 is an echocardiographic tracing from a dog in which one suture was inserted in the subepicardial range $(21 \%$ of the transmural thickness from the transducer-epicardial interface) and one suture was placed in the midwall range $(53 \%$ of transmural thickness). As the left panel illustrates, the deeper myocardial layers contributed progressively more to transmural thickening during control than the superficial layers. The right panel illustrates changes in

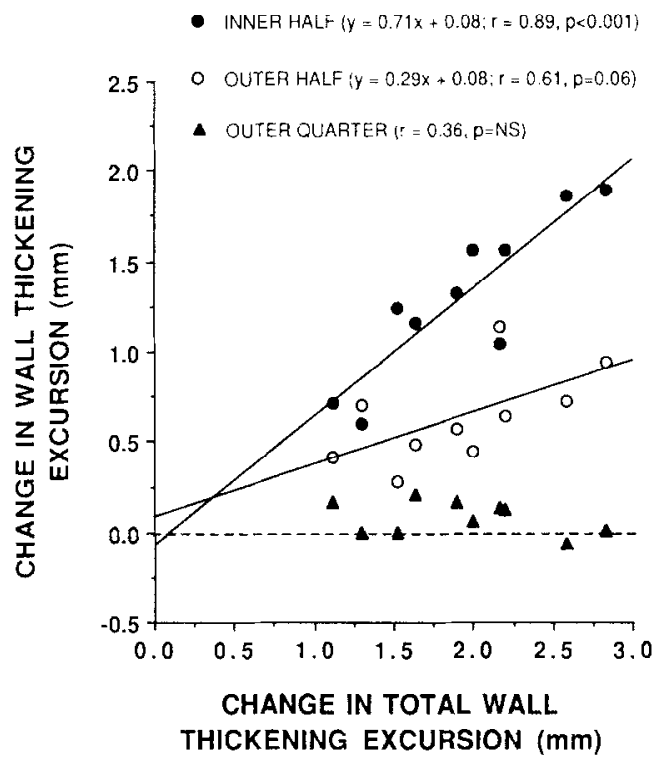

Fig. 3. Regional differences in thickening plotted as a function of the difference in transmural thickening. Although there was a significant linear relationship between transmural and subendocardial function $(\mathrm{y}=0.49+1.10 \mathrm{x}$; $r=0.89 ; p<0.001)$, and transmural and midwall function tended to correlate $(\mathrm{y}=1.10+1.30 \mathrm{x} ; r=0.61 ; p=0.059)$, there was no relationship between subepicardial and transmural function ( $\mathrm{y}=2.10-2.20 \mathrm{x} ; r=0.36 ; p=\mathrm{NS})$. There was also no statistical relationship between EPI function and MID or ENDO function during PCO. WE, Wall excursion; $P C O$, partial coronary occlusion; $E P I$, subepicardial suture data; $M I D$, midwall suture data; $E N D O$, transmural minus midwall data.

regional wall thickening produced by a coronary stenosis that resulted in approximately a $50 \%$ decrease in transmural thickening. Although there was a significant decrease in transmural excursion, regional wall excursion, as depicted by suture movement, showed differing degrees of dysfunction. Subepicardial thickening was reduced only minimally $(\sim 10 \%)$ in this example. These changes in regional wall motion reflected the nonuniform distribution of coronary blood flow during partial stenosis. That is, blood flow decreased by $60 \%, 50 \%$, and $14 \%$ in the subendocardium, midwall, and subepicardium, respectively, in this dog.

Table II summarizes the echocardiographic data for the 10 dogs with partial coronary occlusion lasting approximately 5 minutes. The data during baseline conditions were characterized by a nonuniform pattern, similar to that in previous reports. ${ }^{1-3}$ During baseline conditions, the outer one quarter of the myocardial wall contributed $15.6 \pm 2.1 \%$ to total transmural wall thickening, the outer half of the wall contributed $33.9 \pm 3.6 \%$ to total thickening, and the inner half contributed the remaining $66.1 \pm 3.6 \%$. 


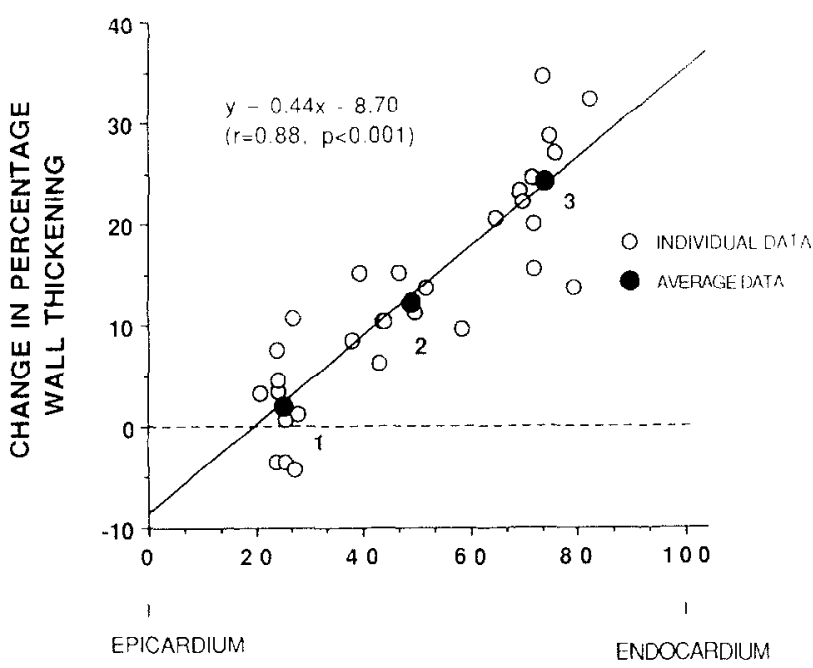

DEPTH OF WALL THICKENING MEASUREMENT

Fig. 4. Regional differences in percent wall excursion expressed as a function of tissue depth. 1, Average data from the subepicardial sutures; 2 , average data from the midwall sutures; 3 , average data derived by subtracting midwall data from transmural data.

Fig. 2 illustrates the changes in subepicardial (outer quarter), midwall (outer half), inner half, and total wall systolic excursion during partial coronary occlusion. Although all categories of wall thickening displayed significant reductions in systolic excursion, the least affected was the subepicardial quarter. After partial coronary occlusion was produced, total wall thickening decreased from a baseline level of $5.06 \pm 0.31 \mathrm{~mm}$ to $3.13 \pm 0.22 \mathrm{~mm}(p<0.001)$, consistent with the experimental design. Simultaneously measured subepicardial (outer quarter) wall thickening displayed a slight, but statistically significant decrease in systolic excursion, from $0.77 \pm 0.08 \mathrm{~mm}$ to $0.69 \pm 0.08 \mathrm{~mm}(p<0.05)$. There was no significant decrease, however, in subepicardial percentage wall thickening (baseline: $30.8 \pm 3.7 \%$; partial coronary occlusion: $28.7 \pm 3.6 \% ; p=\mathrm{NS}$ ). Midwall (or outer half) systolic excursion decreased substantially from $1.66 \pm 0.14 \mathrm{~mm}$ to $1.03 \pm 0.09 \mathrm{~mm}(p<0.001)$, and inner wall thickening decreased from $3.40 \mathrm{~mm}$ to $2.10 \mathrm{~mm}(p<0.001)$. The fractional contribution of the outer quarter of the wall to total wall thickening increased significantly during nontransmural ischemia to $23.0 \pm 3.5 \% \quad(p<0.05)$. There was no change, however, in the fractional contribution of the inner and outer halves of the wall during nontransmural ischemia, compared with baseline conditions.

Shown in Fig. 3 is the relationship between the change in wall excursion by layer (outer quarter, outer half, inner half) and the change in total or

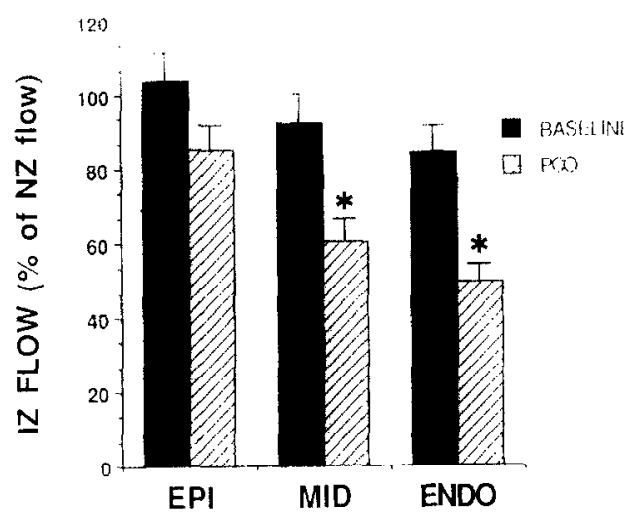

Fig. 5. Regional ischemic zone (IZ) blood flow expressed as a percent of normal zone $(N Z)$ blood flow during partial coronary occlusion $(P C O) .{ }^{*} p<0.05$ from control value. $E P I$, Subepicardium; $M I D$, midwall; ENDO, subendocardium.

transmural wall thickening. The changes in subepicardial excursion were small and did not correlate with the changes in transmural excursion $(r=-0.36$; $p=\mathrm{NS}$ ), suggesting that the small changes in subepicardial thickening during nontransmural ischemia were largely unrelated to the overall wall motion change. Wall thickening in the outer half of the wall (midwall) tended to correlate better with transmural dysfunction $(r=0.61)$, but the relationship was of marginal statistical significance $(p=0.059)$. Decreases in inner wall thickening, however, correlated closely $(r=0.89)$ and in a highly significant manner $(p<0.001)$ with transmural reductions in systolic excursion.

Fig. 4 illustrates the relationship between the degree of wall thickening impairment (measured as the change in percentage wall thickening) and the depth of the wall thickening measurement from the epicardial surface. The degree of dysfunction is augmented linearly $(r=0.88 ; p<0.001)$ with increasing tissue depth, further demonstrating that the subendocardial half of the wall is the portion most seriously affected by acute nontransmural ischemia while the subepicardial quarter is the least affected.

Normalized myocardial blood flow data are presented in Fig. 5. Blood flow to each region (epi, mid, endo) in the ischemic zone was normalized by expressing it as a percentage of simultaneously measured blood flow to the same region in the nonischemic or control area. ${ }^{13,17-20}$ Myocardial blood flow data were available from 8 of the 10 dogs; values from two of the dogs were discarded because of technical problems with the microsphere injections. Consistent with numerous reports in the literature, coronary inflow restriction reduced flow most dramati- 


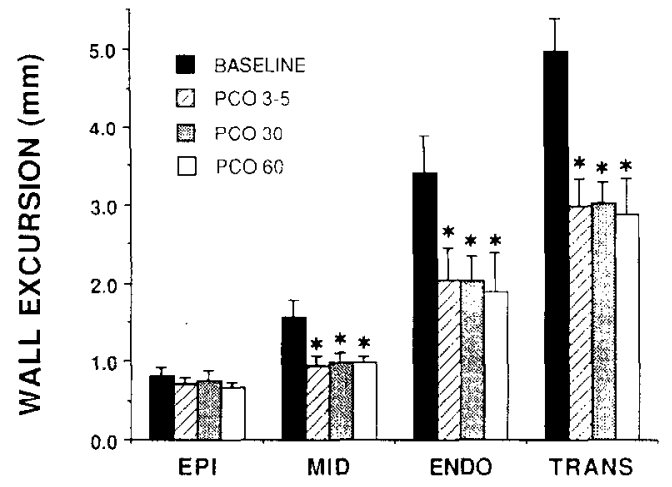

Fig. 6. Regional and transmural wall excursion data during baseline, 3 to 5,30 , and 60 minutes of partial coronary occlusion ( $P C O$ ). EPI, Subepicardium; $M I D$, midwall; $E N D O$, transmural minus midwall thickening. ${ }^{*} p<0.05$ from control value (repeated measures ANOVA).

cally in the subendocardial third of the wall ( $50 \pm 5 \%$ of control area flow), significantly reduced flow in the midmyocardial third ( $61 \pm 6 \%$ of control area flow), and produced only a slight, nonsignificant decrease ( $85 \pm 7 \%$ of control flow) in the subepicardial third. Thus the pattern of change in wall thickening tended to follow the change in the pattern of myocardial perfusion. It is not surprising that the inner layers of the wall displayed the greatest functional decrease, given that perfusion was restricted to the greatest degree in the inner part of the left ventricular wall. In contrast with other studies that demonstrated marked dissociation between subepicardial flow and function, subepicardial (outer quarter) wall thickening was only minimally reduced in the normally perfused outer layers in the present study.

In five additional dogs, heart rate was controlled by atrial pacing and the partial coronary occlusion was maintained for 60 minutes to determine if the changes observed with more acute conditions would remain stable. Wall thickening data for this group are summarized in Fig. 6. Partial coronary occlusion resulted in the same pattern of change in wall thickening across the left ventricular wall. Subendocardial (inner half) and midwall (outer half) wall thickening were significantly and consistently reduced from baseline throughout the period of ischemia. Subepicardial (outer quarter) wall thickening displayed a small, nonsignificant decrease that was also maintained through the 60 -minute ischemic period. The decrease in subepicardial thickening averaged approximately $10 \%$ from baseline but was not statistically significant in the paced, 60-minute ischemia group, probably because the sample size was smaller than in the 5-minute ischemia group. Normalized

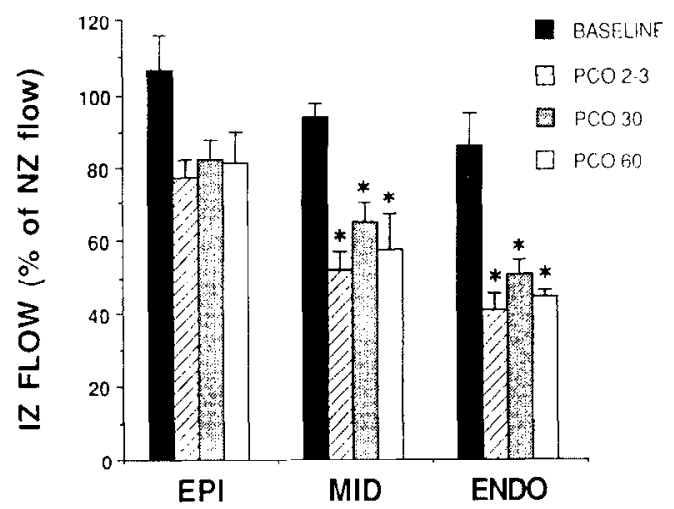

Fig. 7. Regional ischemic zone (IZ) blood flow expressed as a percent of normal zone $(N Z)$ blood flow during baseline, 2 to 3,30 , and 60 minutes of partial coronary occlusion (PCO). EPI, Subepicardium; MID, midwall; ENDO, subendocardium. ${ }^{*} p<0.05$ from control value (repeated measures ANOVA).

blood flow data from the 60-minute ischemia group are summarized in Fig. 7. These data were also consistent across time and were similar to the acute changes produced in the 5-minute ischemia group. The pattern of functional changes in this subgroup of animals also paralleled the pattern of perfusion, and there was little evidence that subepicardial (outer quarter) wall motion was constrained by transmural tethering to the dysfunctional subendocardial layers.

\section{DISCUSSION}

Epicardial echocardiography has been used in several studies to measure regional systolic wall thickening. ${ }^{21-25}$ The use of epicardial echocardiography to measure wall thickness avoids the potential problem of ultrasonic crystal alignment with respect to regional fiber orientation. ${ }^{27}$ In addition, the echosuture technique is somewhat less traumatic than sonomicrometry, ${ }^{1}$ which may be a particularly severe problem in the epicardium, which is prone to trauma and distortion from crystal insertion. ${ }^{11}$

Epicardial echocardiography was used in this study to directly evaluate regional wall thickening dynamics during baseline and nontransmural ischemic conditions to address the problem of transmural tethering. During baseline conditions, the anterior free wall of the left ventricle exhibited nonuniform wall thickening. The outer half of the wall contributed $34 \%$ to transmural thickening and the inner half contributed $66 \%$, results that are consistent with those of our previous study ${ }^{1}$ and with those of other investigators. ${ }^{2-7}$

Partial coronary stenosis produced the characteristic maldistribution of myocardial blood flow associated with moderate coronary inflow restric- 
tion. $8,9.15,26$ Subepicardial blood flow remained nearly normal, whereas midmyocardial and subendocardial flows were significantly reduced, resulting in a reduction of transmural wall thickening that averaged approximately $40 \%$ from baseline. Large changes in wall thickening were also observed in the outer and inner halves of the left ventricular wall (Table II, Fig. 2). The nonuniform changes in regional myocardial blood flow and thickening (Figs. 6 and 7) were consistent over time in the five dogs that were paced during 60 minutes of partial coronary obstruction, supporting the view that the regional thickening data obtained after 5 minutes of coronary inflow restriction represented steady-state conditions.

The crucial measurement in the present study to evaluate the issue of transmural tethering was that of subepicardial (or outer quarter) wall thickening. The fractional contribution of the outer quarter to total wall thickening during baseline conditions averaged approximately $15 \%$, a result in line with simple theoretical predictions ${ }^{3}$ and in accord with the previous findings cited above. Subepicardial wall thickening during nontransmural ischemia was reduced approximately $10 \%$, a degree of reduction that was statistically significant in the 5 -minute ischemia group but not statistically significant in the 60-minute ischemia group. The change was small and at the limits of the spatial resolution of the epicardial echocardiographic technique $(0.15 \mathrm{~mm})$. Accordingly, we propose that the biologic significance of the statistical change in subepicardial thickening observed was trivial. We attribute the small decrease in subepicardial thickening during coronary inflow restriction largely to the trend for a small decrease in regional blood flow and not to a transmural tethering effect, since there was no significant correlation between the degree of subepicardial dysfunction and inner wall or transmural dysfunction during partial coronary stenosis (Fig. 3). This conclusion contrasts with the findings of other investigators, ${ }^{10,11,15}$ who have suggested that a significant transmural tethering effect influences outer wall function in the setting of nontransmural ischemia.

Direct comparisons of our epicardial echocardiographic findings with those based on the use of sonomicrometers or other techniques are complicated by fundamental differences in the various methods used to measure regional function. Indeed, significant differences are evident even among the studies using sonomicrometers to assess regional myocardial function during nontransmural ischemia. ${ }^{10,12,14,27}$ For example, Weintraub et al. ${ }^{10} \mathrm{ob}$ served that subepicardial segment shortening in the anterior wall tended to parallel the degree of subendocardial dysfunction during graded reductions in coronary flow despite apparently normal subepicardial blood flow. They were the first to propose that subepicardial wall motion is constrained by transmural tethering to dysfunctional subendocardial layers. Hattori et al. ${ }^{11}$ also found that both anterior wall long-axis and short-axis segment shortening in the subepicardium tended to decrease in parallel with shortening in the subendocardium during graded reductions in perfusion pressure.

Gallagher et al. ${ }^{12}$ also found a good association between changes in circumferential epicardial shortening and subendocardial shortening during partial circumflex artery stenosis despite maintenance of subepicardial blood flow. Epicardial segment shortening parallel to surface fiber orientation in the posterior wall of the left ventricle, however, remained at normal levels until subepicardial blood flow was reduced by more severe coronary stenosis. ${ }^{12}$ In a later study, Gallagher et al. ${ }^{27}$ confirmed their initial findings but also observed that there were significant regional differences in the subepicardial functional response to subendocardial ischemia. Epicardial shortening in the anterior wall (parallel to surface fiber orientation) decreased modestly but significantly, although subepicardial blood flow was at normal levels. ${ }^{27}$

Bertha and Folts ${ }^{14}$ found that the onset of shortening dysfunction in the subendocardium preceded that in the subepicardium during continuous, gradual coronary flow reductions produced by thrombus formation in the circumflex artery. Some degree of subepicardial shortening was maintained at the lowest blood flow levels despite holosystolic bulging in the subendocardium. They concluded that the epicardium is not tethered to the endocardium. Although they did not measure myocardial blood flow to each "layer" of the wall, subendocardial shortening was more sensitive to reductions in total circumflex flow than was subepicardial function. ${ }^{14}$ Our results with epicardial echocardiography demonstrate that subepicardial function, determined by wall thickening, showed little change despite significant subendocardial dysfunction produced by moderate coronary inflow restriction. These results are similar to those of others in which subepicardial function was assessed by segmental shortening parallel to surface fiber orientation. ${ }^{12,14,27}$

Although subepicardial function was minimally affected by partial coronary stenosis in the present study, the relative importance of maintaining epicardial function in terms of transmural thickening appeared to be small. Even without significant teth- 
ering constraints restricting outer wall function (as the present data suggest), a large reduction in transmural thickening will be evident when the inner portion of the wall is ischemic simply because significantly more thickening (or segment shortening) occurs in this part of the wall. ${ }^{1-3}$ The maintenance of epicardial function, however, may prove useful to limit the degree of systolic bulging that occurs with more severe ischemia than that produced in the present study. ${ }^{12,14}$

\section{REFERENCES}

1. Myers JH, Sterling MC, Buda M, Gallagher KP. Direct measurement of inner and outer wall thickening dynamics with epicardial echocardiography. Circulation 1986;74:164-72.

2. Sabbah HN, Marzilli M, Stein PD. The relative role of subendocardium and subepicardium in left ventricular mechanics. Am J Physiol 1981;240:H920-6.

3. Gallagher KP, Osakada G, Matsuzaki M, Miller M, Kemper WS, Ross J Jr. Nonuniformity of inner and outer systolic wall thickening in conscious dogs. Am J Physiol 1985;249:H241-8.

4. Zhu WX, Myers ML, Hartley CJ, Roberts R, Bolli R. Validation of a single crystal for measurement of transmural and epicardial thickening. Am J Physiol 1986;251:H1045-55.

5. Homans DC, Sublett E, Lindstrom P, Nesbitt T, Bache RJ. Subendocardial and subepicardial wall thickening during ischemia in exercising dogs. Circulation 1988;78:1267-76.

6. Waldman LK, Fung YC, Covell JW. Transmural myocardial deformation in the canine left ventricle. Normal in vivo threedimensional finite strains. Circ Res 1985;57:152-63.

7. Hittinger L, Shannon RP, Kohin S, Manders WT, Kelly P Vatner SF. Exercise induced subendocardial dysfunction in dogs with left ventricular hypertrophy. Circ Res 1990;66:329. 43.

8. Bache RJ, McHale PA, Greenfield JC. Transmural myocardial perfusion during restricted coronary inflow in the awake dog. Am J Physiol 1977;232:H645-51.

9. Hoffman JIE. Determinants and prediction of transmural myocardial perfusion. Circulation 1978;58:381-91.

10. Weintraub WS, Hattori S, Agarwal JB, Bodenheimer MM, Banka VS, Helfant RH. The relationship between myocardial blood flow and the contraction by myocardial layer in the canine left ventricle during ischemia. Circ Res 1981;48:430-8.

11. Hattori S, Weintrab WS, Agarwal JB, Bodenheimer MM, Banka VS, Helfant RH. Contrasting ischemic contraction patterns by zone and layer in canine myocardium. Am J Physiol 1982;243:H852-5.

12. Gallagher KP, Osakada G, Hess OM, Koziol J, Kemper WS, Ross J Jr. Subepicardial segmental function during coronary stenosis and the role of myocardial fiber orientation. Circ Res 1982;50:352-9.
13. Gallagher KP, Matsuzaki M, Koziol JA, Kemper WS, Ross J Jr. Regional myocardial perfusion and wall thickening during ischemia in conscious dogs. Am .J Physiol 1984;247:H727-38.

14. Bertha BG, Folts JD. Subendocardial and subepicardial segmental function changes in the dog heart due to gradual coronary flow reduction by an acutely developing thrombus. Cardiovasc Res 1985;19:495-506.

15. Prinzen FW, Arts T, Van der Wusse GJ, Coumans WA, Reneman RS. Gradients in fiber shortening and metabolism across ischemic left ventricular wall. Am J Physiol 1986;250:H255-64.

16. Heymann MA, Payne BD, Hoffman JIE, Rudolf AM. Blood flow measurements with radionuclide-labeled particles. Prog Cardiovasc Dis 1977;21:55-79.

17. Vatner SF. Correlation between acute reductions in myocardial blood flow and function in conscious dogs. Circ Res 1980;47:201-7.

18. Matsuzaki M, Gallagher KP, Kemper S, White F, Ross J Jr. Sustained regional dysfunction produced by prolonged coronary stenosis: gradual recovery after reperfusion. Circulation $1983 ; 68: 170-82$.

19. Canty JM Jr. Coronary perfusion-function and steady-state pressure-flow relations during autoregulation in the anesthetized dog. Circ Res 1988;63:821-36.

20. Miura T, Yoshida S, limura O, Downey JM. Dobutamine modifies myocardial infarct size through supply-demand balance. Am J Physiol 1988;254:H855-61.

21. Myerowitz PD, Griffith J, Roberts AJ, Harrison LH, Henery WL, McIntosh CL. Long-term canine model for echocardiography. Am J Cardiol 1974;34:72-4.

22. Kerber RE, Marcus ML, Ehrhardt J, Wilson R, Abboud FM Correlation between echocardiographically demonstrated seg mental dyskinesis and regional myocardial perfusion. Circulation 1975;52:1097-104.

23. Kerber RE, Marcus ML, Ehrhardt J, Wilson R, Abboud FM. Effect of acute coronary occlusion on the motion and perfusion of the normal and ischemic interventricular septum: an experimental echocardiographic study. Circulation 1976; 54:928-35.

24. Gaasch WH, Barnard SA. The effect of acute changes in coronary blood flow on left ventricular end-diastolic wall thickness. Circulation 1977;56:593-8.

25. Egeblad H, Hauno S, Amtorp O. Regional dynamic behaviour of the canine myocardium following brief ischemia: wall thickness, wall thickening and hlood flow. Cardiovasc Res $1982 ; 16: 249-55$

26. Bache R, Schwartz JS. Effect of perfusion pressure distal to a coronary stenosis on transmural myocardial blood flow. Circulation 1982;65:928-35.

27. Gallagher KP, Sterling MC, Choy M, Szpunar CA, Gerren RA, Botham MJ, Lemmer JH. Dissociation between epicardial and transmural function during acute myocardial ischemia. Circulation 1985;71:1279-91. 